\title{
我国北部冬麦区小麦区域试验重复次数和试点数量的优化设计
}

\author{
张 毅 ${ }^{1}$ 许乃银 $^{2}$ 郭利磊 $^{1}$ 杨子光 $^{3}$ 张笑晴 $^{1}$ 杨晓妮 $^{2}$
}

${ }^{1}$ 全国农业技术推广服务中心, 北京 $100125 ;^{2}$ 江苏省农业科学院, 江苏南京 $210014 ;{ }^{3}$ 洛阳农林科学院, 河南洛阳 471022

摘 要: 农作物品种区域试验重复次数和试点数量的合理配置有利于提高试验的成本效率和品种选择效率。本研究 分析了 2010-2019 年期间北部冬麦区小麦品种区域试验的重复次数和试点数量设置的合理性, 依据小麦品种试验的 信噪比和遗传力水平随重复次数和试点数量的变化规律, 提出了重复次数和试点数量的优化设计方案。结果表明: (1) 北部冬麦区小麦单点试验的遗传力平均达到 0.87 , 需要的重复次数平均值仅为 1.4 , 说明 3 次重复可以充分保证试验 精确度的需求。(2)北部冬麦区水地组和旱地组小麦区域试验达到 0.75 的遗传力水平时, 需要的试点数量分别为 11 个和 13 个, 目前有效试点数量分别约为 11 个和 8 个, 分别达到 0.75 和 0.60 的遗传力水平。(3)小麦品种区域试验结 果对品种的审定和应用十分重要, 而每年都可能有少数试验点因为各种异常情况而报废, 为保证试验结果的可靠性, 可按 $H=0.75$ 的水平需求安排试验点数量和重复次数, 即重复次数可保持当前的 3 次; 水地组的试点数量可保持在 11 个左右; 旱地组可将试点增加到 13 个; 如要将遗传力提高到 0.80 的水平, 则需约 16 个试点。

关键词: 小麦(Triticum aestivum L.); 区域试验; 遗传力; 信噪比; 重复次数; 试点数量

\section{Optimization of test location number and replicate frequency in regional winter wheat variety trials in northern winter wheat region in China}

\author{
ZHANG Yi ${ }^{1}$, XU Nai-Yin ${ }^{2}$, GUO Li-Lei ${ }^{1}$, YANG Zi-Guang ${ }^{3}$, ZHANG Xiao-Qing ${ }^{1}$, and YANG Xiao-Ni ${ }^{2}$ \\ ${ }^{1}$ National Extension and Service Center of Agricultural Technology, Beijing 100125, China; ${ }^{2}$ Jiangsu Academy of Agricultural Sciences, Nanjing \\ 210014, Jiangsu, China; ${ }^{3}$ Luoyang Academy of Agriculture and Forestry Sciences, Luoyang 471022, Henan, China
}

\begin{abstract}
The rational allocation of the replicate frequency and test location number in regional crop trials are highly beneficial to the enhancement of both trial cost efficiency and new cultivar selection efficiency. The rationality of test location number and replicate frequency for national wheat regional trials in the Northern winter wheat region (NWWR) in China was evaluated by using historical experimental datasets during the last 10 years according to the dynamics of trial heritability and noise-signal quotient $(Q)$ with the increase of test locations and replicates within trials in 2010-2019, proposing an optimal design scheme of the replicate frequency and test location number for the wheat planting region. The result indicated three replicates at current achieve 0.87 of the averaged within-trial heritability of single-trials in NWWR, and only 1.4 times of replicates on average was needed to achieve 0.75 of within-trial heritability, so three replicates were obviously sufficient to maintain enough test accuracy. The needed test location number to achieve 0.75 of cross-trial heritability was estimated as 11 for the irrigated group and 13 for the rainfed group in the one-year multi-locational trials in NWWR, while in the current wheat trials that was about 11 and 8 effective test locations, achieving a heritability level of 0.75 and 0.60 , respectively. The irrigated group was exactly meet the trial accuracy requirement $(H=0.75)$, while the rainfed group was somewhat insufficient. Considering the importance of the regional wheat trials in recommending new varieties for registration and production, and the possible trial cancellation due to various abnormalities, the practical management should set a heritability level around 0.75 , and maintain three replicates, with around 11 test locations for the irrigated group, and 13 locations for the rain-fed group. In order to increase heritability to the level of 0.80 , about 16 test locations should be required.
\end{abstract}

Keywords: wheat (Triticum aestivum L.); regional trial; heritability; noise-signal quotient; number of replicate; number of test location 
小麦(Triticum aestivum L.)是我国第三大粮食作 物, 种植面积仅次于玉米和水稻。北方冬麦区主要 包括河北大部、河南、山东、山西南部、江苏和安 徽北部、陕西和甘肃部分地区，是我国小麦主要产 区，种植面积约占全国小麦总面积的 $70 \%$ ，总产占 $75 \%$ 左右 ${ }^{[1]}$ 。北部冬麦区主要包括辽宁南端的营口和 大连市; 河北省境内长城以南的廊坊、保定、沧州、 唐山和秦皇岛市全部; 京、津两市全部; 山西省朔州 以南阳泉、太原、晋中、长治和吕梁等市全部和临 汾市北部地区; 陕西省延安市全部、榆林长城以南 大部，咸阳、宝鸡和铜川市部分县; 甘肃省陇东庆阳 市全部和平凉市的部分县 ${ }^{[2]}$ 。北部冬麦区作为北方 冬麦区的重要产区，提供了其中 $22 \%$ 以上的产量 ${ }^{[3]}$ 。 加强适应于该区域生态条件的小麦新品种选育和推 广应用对保障小麦生产水平和我国粮食安全具有重 要意义。小麦品种区域试验在多环境条件下对新品 种的丰产性、稳产性、适应性、抗逆性、品质等性 状进行测试与综合评价, 对促进我国小麦品种更新 和保障小麦生产水平起着重要的作用 ${ }^{[4]}$ 。区域试验 的可靠性主要决定于试验效率, 而试验效率又决定 于试验遗传力 ${ }^{[5-6]}$ 。遗传力是遗传方差占表型方差的 比率 ${ }^{[7]}$, 区域试验的遗传力越高, 对品种的选择效 率就越高 ${ }^{[8]}$ 。遗传力随着试验重复次数和试点数量 的增加速率呈先快后慢的“边际收益递减”规律 ${ }^{[6]}$ 。因 此, 如何合理设置试验试点数量和重复次数, 既可 保持可靠的遗传力水平和试验效率, 又可合理利用 有限的人力、物力和财力资源, 是优化区域试验设 置方案中亟待解决的问题。

严威凯等 ${ }^{[8]}$ 利用区域试验重复次数和试点数量 与遗传力的函数关系, 估算出燕麦品种试验在特定 误差水平下不同遗传力水平需要的试验重复次数和 试点数量, 并指出通过增加试点数和重复次数可以 快速提高遗传力的上限为 $75 \%$, 其后遗传力提高的 速率急剧降低, 故认为试验遗传力达到 0.75 水平时 需要的重复次数和试点数量是较适宜的数量。许乃 银等 ${ }^{[5]}$ 对国家棉花区试的遗传力水平及其随着试点 数量增加的规律进行了定量化分析, 并提出针对各 主产棉区的区域试验设置优化方案。关于小麦品种 区域试验适宜重复次数和试点数量的相关研究尚未 见报道。本研究的目的是综合利用重复次数和试点 数量的估计方法, 全面分析和评价 2010-2019 年期 间我国北部冬麦区国家小麦品种试验重复次数和试 点数量设置的科学性和优化方案, 为国家小麦区域
试验的优化资源配置提供依据, 并为其他作物品种 区域试验的优化设置提供参考。

\section{1 材料与方法}

\section{1 数据来源}

2010-2019 年期间北部冬麦区水地组和旱地组 小麦品种试验设置的试点数量和参试品种数详见表 1 。 所有试验均采用随机区组排列, 重复 3 次, 小区面 积 12 14 $\mathrm{m}^{2}$ 。2009-2010 年度(简称为“2010 年”，下 同)水地组区域试验以“京冬 8 号”为对照, 以“中麦 175”辅助对照品种。其余年份水地组和旱地组小麦 品种试验分别以中麦 175 和“长 6878 ”为对照品种。 水地组常年在北京市的昌平和顺义; 天津市的武清 和宝坻; 河北省的保定、遵化、㴒南和固安; 山西省 的太原、屯留、介休等地共设置约 11 个试点(2015 年前在新疆设有阿拉尔试点)。旱地组常年在山西省 的高平、长子、长治和平凉; 甘肃省的庄浪、定西、 镇原和庆阳; 宁夏的固原、隆德、彭阳等地共设置 约 11 个试点。田间栽培管理按当地习惯和区域试验 方案要求进行。本研究分别采用 2010-2019 年期间 北部冬麦区小麦品种试验的 196 个单年单点试验(其 中, 水地组 112 个, 旱地组 84 个)和 20 组一年多点 试验的产量数据分析试验重复次数和试点数量与遗 传力的关系, 估算在特定遗传力水平下对重复次数 和试点数量的需求。

\section{2 统计分析}

采用数据分析和管理系统 GGEbiplot Pattern Explorer ${ }^{[9]}$ 对 2010-2019年期间北部冬麦区小麦品 种试验中196个单点试验分别进行方差分析, 得出遗 传方差和误差方差, 再依据 Yan 等 $^{[6,8]}$ 和许乃银等 ${ }^{[5]}$ 介绍的方法分别计算单点试验的信噪比、遗传力及 遗传力为 0.75 时需要的试验重复次数, 并根据196次 单点试验在不同重复次数需求范围内的累积频数 (cumulative frequency, C f ) 分布规律, 初步分析小麦 品种区域试验的适宜重复次数。其次, 对 20 组一年 多点试验分别进行方差分析, 得出遗传方差、品种 与试点互作方差和误差方差, 计算一年多点试验的 信噪比、遗传力及遗传力为 0.75 时需要的试点数量, 分别估计水地组和旱地组试验对试点数量的需求。 然后, 依据水地组和旱地组小麦品种试验的多次单 点试验平均信噪比 $\left(Q_{\mathrm{r}}\right)$ 和一年多点试验平均信噪比 $\left(Q_{\mathrm{e}}\right)$ 与重复次数、试点数及遗传力的关系式, 对不同 重复次数和试点数量条件下的遗传力水平进行定量 
分析。最后, 依据遗传力与信噪比、重复次数和试点 数的关系式, 在设定区间内拟合遗传力增量与重复 次数和试点数量增量的响应曲线，从而提出小麦品 种试验重复次数和试点数量设置的优化方案。单年单 点和一年多点区域试验的遗传力及其增量、信噪比、 重复次数和试点数量的计算公式如下 ${ }^{[5-6]}$ :

单年单点试验的遗传力 $H=V_{\mathrm{g}} /\left(V_{\mathrm{g}}+V_{\mathrm{e}} / n_{\mathrm{r}}\right)$

单年单点试验的信噪比 $Q_{\mathrm{r}}=V_{\mathrm{e}} / V_{\mathrm{g}}$

单年单点试验所需的重复数 $N_{\mathrm{r}}=\left(V_{\mathrm{e}} / V_{\mathrm{g}}\right) \times H /$ $(1-H)=Q_{\mathrm{r}} \times H /(1-H)$

单年单点试验遗传力与重复次数及信噪比的关 系式 $H=N_{\mathrm{r}} /\left(N_{\mathrm{r}}+Q_{\mathrm{r}}\right)$

单年单点试验遗传力增量 $\Delta H=\left[N_{\mathrm{r}} /\left(N_{\mathrm{r}}+Q_{\mathrm{r}}\right)\right]-$ $\left[N_{(\mathrm{r}-\Delta \mathrm{r})} /\left(N_{(\mathrm{r}-\Delta \mathrm{r})}+Q_{\mathrm{r}}\right)\right]$

一年多点试验的遗传力 $H=V_{\mathrm{g}} /\left(V_{\mathrm{g}}+V_{\mathrm{ge}} / n_{\mathrm{e}}+\right.$
$\left.V_{\mathrm{e}} / n_{\mathrm{e}} n_{\mathrm{r}}\right)$

一年多点试验的信噪比 $Q_{e}=\left(V_{\mathrm{ge}}+V_{\mathrm{e}} / n_{\mathrm{e}}\right) / V_{\mathrm{g}}$ (7)

一年多点试验所需的试点数 $N_{\mathrm{e}}=$ $\left[\left(V_{\mathrm{ge}}+V_{\mathrm{e}} / n_{\mathrm{e}}\right) / V_{\mathrm{g}}\right] \times H /(1-H)=Q_{\mathrm{e}} \times H /(1-H)$

一年多点试验遗传力与试点数及信噪比的关系

式 $H=N_{\mathrm{e}} /\left(N_{\mathrm{e}}+Q_{\mathrm{e}}\right)$

一年多点试验遗传力增量 $\Delta H=\left[N_{\mathrm{e}} /\left(N_{\mathrm{e}}+Q_{\mathrm{e}}\right)\right]-$ $\left[N_{(\mathrm{e}-\Delta \mathrm{e})} /\left(N_{(\mathrm{e}-\Delta \mathrm{e})}+Q_{\mathrm{e}}\right)\right]$

式中, $H$ 为遗传力, $V_{\mathrm{g}}$ 为遗传方差, $V_{\mathrm{e}}$ 为误差方差, $n_{\mathrm{r}}$ 为重复数, $Q_{\mathrm{r}}$ 为单点试验信噪比, $N_{\mathrm{r}}$ 为单点试验需要 的重复数, $V_{\mathrm{ge}}$ 为品种与试点互作方差, $n_{\mathrm{e}}$ 为试点数量, $Q_{\mathrm{e}}$ 为一年多点试验的信噪比, $N_{\mathrm{e}}$ 为一年多点试验需 要的试点数量, $\Delta H$ 为试验遗传力增量, $\Delta \mathrm{r}$ 和 $\Delta \mathrm{e}$ 分别 为单位重复数和试点数, $N_{(\mathrm{r}-\Delta \mathrm{r})}$ 和 $N_{(\mathrm{e}-\Delta \mathrm{e})}$ 分别为在 $N_{\mathrm{r}}$ 和 $N_{\mathrm{e}}$ 基础上减少一个单位的重复数和试点数。

表 $12010-2019$ 年我国北部冬麦区国家小麦品种区域试验组数、品种数和产量统计表

Table 1 Summary statistics of trial yield grand mean, number of cultivars and locations used in regional winter wheat variety trials in Northern winter wheat region from 2010 to 2019

\begin{tabular}{|c|c|c|c|c|c|c|c|c|}
\hline \multirow{2}{*}{$\begin{array}{l}\text { 年度 } \\
\text { Year }\end{array}$} & \multicolumn{3}{|c|}{ 水地组 } & \multicolumn{3}{|c|}{$\begin{array}{c}\text { 旱地组 } \\
\text { Rainfed group }\end{array}$} & \multicolumn{2}{|c|}{$\begin{array}{c}\text { 北部冬麦区 } \\
\text { Northern winter wheat region }\end{array}$} \\
\hline & $\begin{array}{l}\text { 试点数 } \\
\text { Site }\end{array}$ & $\begin{array}{l}\text { 品种数 } \\
\text { Cultivar }\end{array}$ & $\begin{array}{c}\text { 产量 } \\
\text { Yield }\left(\mathrm{kg} \mathrm{hm}^{-2}\right) \\
\end{array}$ & $\begin{array}{l}\text { 试点数 } \\
\text { Site }\end{array}$ & $\begin{array}{l}\text { 品种数 } \\
\text { Cultivar }\end{array}$ & $\begin{array}{c}\text { 产量 } \\
\text { Yield }\left(\mathrm{kg} \mathrm{hm}^{-2}\right) \\
\end{array}$ & $\begin{array}{l}\text { 试点数 } \\
\text { Site }\end{array}$ & $\begin{array}{l}\text { 品种数 } \\
\text { Cultivar }\end{array}$ \\
\hline 2009-2010 & 11 & 13 & 6178.1 & 9 & 9 & 3581.0 & 20 & 22 \\
\hline 2010-2011 & 12 & 12 & 6717.5 & 9 & 10 & 3885.5 & 21 & 22 \\
\hline 2011-2012 & 11 & 11 & 6895.7 & 7 & 8 & 5524.9 & 18 & 19 \\
\hline $2012-2013$ & 11 & 14 & 6119.9 & 9 & 10 & 3213.0 & 20 & 24 \\
\hline 2013-2014 & 12 & 14 & 7485.6 & 9 & 10 & 5165.8 & 21 & 24 \\
\hline 2014-2015 & 12 & 10 & 7935.6 & 10 & 13 & 5490.4 & 22 & 23 \\
\hline $2015-2016$ & 11 & 16 & 7996.1 & 7 & 12 & 4737.7 & 18 & 28 \\
\hline 2016-2017 & 10 & 11 & 8536.2 & 9 & 12 & 4417.7 & 19 & 23 \\
\hline 2017-2018 & 11 & 14 & 7153.2 & 9 & 8 & 4719.7 & 20 & 22 \\
\hline 2018-2019 & 11 & 17 & 8367.4 & 6 & 8 & 4679.4 & 17 & 25 \\
\hline 平均 Mean & 11.2 & 13.2 & 7338.5 & 8.4 & 10.0 & 4541.5 & 19.6 & 23.2 \\
\hline 合计 Total & 112 & 132 & - & 84 & 100 & - & 196 & 232 \\
\hline
\end{tabular}

\section{2 结果与分析}

2.1 单年单点小麦区域试验的重复次数分析

表 2 表明: (1)在北部冬麦区共 196 次单点试验中, 在保证遗传力达到 0.75 水平下需要 1 次、2 次和 3 次 重复以内的试验累积频率分别约为 $69 \%$ 、83\%和 $88 \%$, 需要 3 4 次重复、4 5 次重复和 5 次以上重复的试验 比例分别约为 $4 \% 、 2 \%$ 和 $7 \%$ 。其中, 水地组和旱地组
需要 3 次重复以内的试验累积频率分别约为 $87 \%$ 和 $89 \%$, 差异不大。可见, 采用 3 次重复可以保证近 $90 \%$ 的单点试验遗传力达到 0.75 的水平, 仅有少数试点因 试验误差偏大等原因导致遗传力偏低; (2)北部冬麦区 共 196 次单点试验需要的重复次数总均值仅为 1.4 次, 其中, 水地组和旱地组需要的重复次数平均都在 2 次以 下, 说明北部冬麦区小麦品种试验采用 3 次重复可以充 分保证试验遗传力和试验效率符合试验质量要求。 
表 2 2010-2019 年北部冬麦区单年单点小麦品种试验在遗传力为 0.75 时所需要重复数的次数分布

Table 2 Frequency distribution of replicates needed to achieve 0.75 of within-trial heritability for single-trials of regional winter wheat variety trials in Northern winter wheat region from 2010 to 2019

\begin{tabular}{|c|c|c|c|c|c|c|c|c|c|c|c|c|c|c|c|}
\hline \multirow{2}{*}{$\begin{array}{c}\text { 需要重复数 } \\
\text { Interval of } \\
N_{\mathrm{r}} \text { needed }\end{array}$} & \multicolumn{5}{|c|}{$\begin{array}{c}\text { 水地组 } \\
\text { Irrigated group }\end{array}$} & \multicolumn{5}{|c|}{$\begin{array}{c}\text { 旱地组 } \\
\text { Rainfed group }\end{array}$} & \multicolumn{5}{|c|}{$\begin{array}{c}\text { 北部冬麦区 } \\
\text { Northern winter wheat region }\end{array}$} \\
\hline & $\mathrm{N}$ & $f(\%)$ & $\mathrm{C} f(\%)$ & $N_{\mathrm{r}}$ & $Q_{\mathrm{r}}$ & $\mathrm{N}$ & $f(\%)$ & $\mathrm{C} f(\%)$ & $N_{\mathrm{r}}$ & $Q_{\mathrm{r}}$ & $\mathrm{N}$ & $f(\%)$ & $\mathrm{C} f(\%)$ & $N_{\mathrm{r}}$ & $Q_{\mathrm{r}}$ \\
\hline$N_{\mathrm{r}} \leq 1$ & 75 & 67.0 & 67.0 & 0.40 & 0.13 & 60 & 71.4 & 71.4 & 0.30 & 0.10 & 135 & 68.9 & 68.9 & 0.36 & 0.12 \\
\hline $1<N_{\mathrm{r}} \leq 2$ & 14 & 12.5 & 79.5 & 1.41 & 0.47 & 14 & 16.7 & 88.1 & 1.34 & 0.45 & 28 & 14.3 & 83.2 & 1.38 & 0.46 \\
\hline $2<N_{\mathrm{r}} \leq 3$ & 8 & 7.1 & 86.6 & 2.34 & 0.78 & 1 & 1.2 & 89.3 & 2.00 & 0.67 & 9 & 4.6 & 87.8 & 2.30 & 0.77 \\
\hline $3<N_{\mathrm{r}} \leq 4$ & 4 & 3.6 & 90.2 & 3.37 & 1.12 & 3 & 3.6 & 92.9 & 3.30 & 1.10 & 7 & 3.6 & 91.3 & 3.34 & 1.11 \\
\hline $4<N_{\mathrm{r}} \leq 5$ & 2 & 1.8 & 92.0 & 4.20 & 1.40 & 1 & 1.2 & 94.1 & 4.37 & 1.46 & 3 & 1.5 & 92.9 & 4.26 & 1.42 \\
\hline$N_{\mathrm{r}}>5$ & 9 & 8.0 & 100.0 & 9.70 & 3.23 & 5 & 6.0 & 100.0 & 8.90 & 2.97 & 14 & 7.1 & 100.0 & 9.41 & 3.14 \\
\hline 总计 Total & 112 & 100.0 & - & 1.59 & 0.53 & 84 & 100.0 & - & 1.17 & 0.39 & 196 & 100.0 & - & 1.41 & 0.46 \\
\hline
\end{tabular}

重复数范围是指在 $H=0.75$ 时所需要的试验重复次数的分布范围。北部冬麦区包括水地组和旱地组。 $\mathrm{N} 、 f(\%) 、 \mathrm{C} f(\%) 、 N_{\mathrm{r}}$ 和 $Q_{\mathrm{r}}$ 分别 为次数、频数、累积频数、需要重复数和信噪比。

Range of $N_{\mathrm{r}}$ means the replicates needed to achieve 0.75 of heritability in half-open intervals. The Northern winter wheat region covers Irrigated region and Rainfed region. $\mathrm{N}, f(\%), \mathrm{C} f(\%), N_{\mathrm{r}}, Q_{\mathrm{r}}$, and $H(\%)$ stand for number of times, frequency, cumulative frequency, replicate needed and noise-signal ratio in each interval, respectively.

\section{2 小麦品种试验适宜重复数和试点数的定量 分析}

依据 2010-2019 年水地组、旱地组及北部冬麦 区小麦品种试验的单点试验平均信噪比 $\left(Q_{\mathrm{r}}\right)$ 和一年 多点试验平均信噪比 $\left(Q_{\mathrm{e}}\right)$ 与重复次数或试点数及遗 传力的关系式，在不同重复次数和试点数量条件下 对遗传力水平的定量分析(图 1 和表 3)表明: (1)试验 遗传力随着重复次数和试点数的增加, 表现为先快 后慢的曲线关系。随着重复次数和试点数的增加, 遗传力在 0.75 以下时提升速度非常快, 为遗传力的 “快速增长期”; 在 0.75 和 0.90 期间提升速率明显减

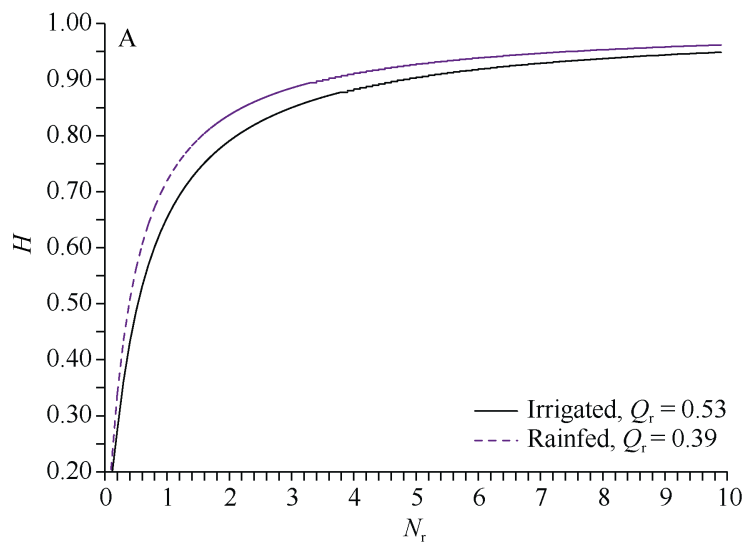

缓，但仍有显著的增量，为遗传力的“缓慢增长期”; 达 0.90 之后, 再增加重复次数或试点数量对遗传力 的提高作用微小，是遗传力的“增长停滞期”。(2)在 遗传力为 0.75 水平下, 水地组、旱地组及北部冬麦 区小麦品种试验需要的重复次数都在 2 次以下; 重 复次数为 3 次的情况下, 水地组、旱地组及北部冬 麦区小麦品种试验的遗传力分别达到了约 $0.85 、 0.88$ 和 0.87 的水平, 已经充分保证了试验的精度要求; 如需将遗传力提高在 0.90 水平下, 则需要约 4 5 次 重复, 其后继续增加重复次数对提高遗传力没有意 义。(3)在遗传力为 0.75 水平下, 水地组、旱地组及

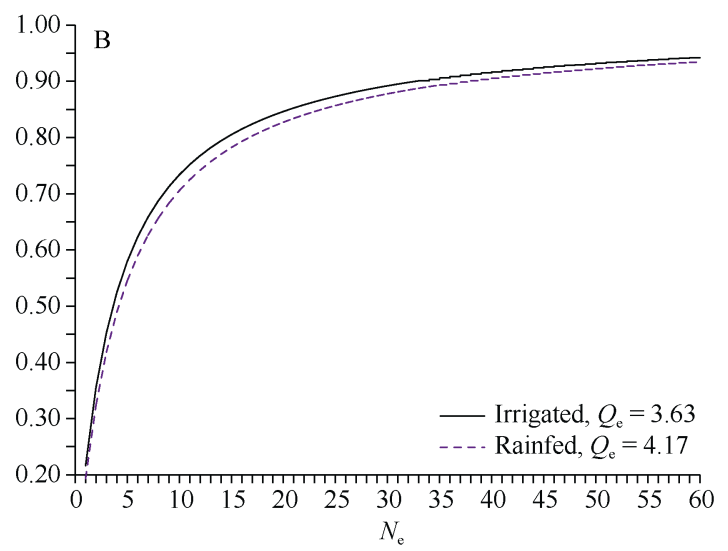

图 1 北部冬麦区小麦品种试验的遗传力 $(H)$ 及其所需重复 $\left(N_{\mathrm{r}}\right)$ 或试点数量 $\left(N_{\mathrm{e}}\right)$ 的关系

Fig. 1 Relationship between heritability $(H)$ and replicate $\left(N_{\mathrm{r}}\right)$ or test location number $\left(N_{\mathrm{e}}\right)$ for regional winter wheat variety trials in Northern winter wheat region Irrigated 和 Rainfed 分别代表北部冬麦区的水地组和旱地组小麦品种试验; $Q_{\mathrm{r}}$ 和 $Q_{\mathrm{e}}$ 分别代表重复数信噪比和试点数信噪比。 Irrigated and Rainfed stand for the irrigated group and rainfed group in winter wheat variety trials of Northern winter wheat region. $Q_{\mathrm{r}}$ and $Q_{\mathrm{e}}$ stand for the noise-signal ratio of replicate and test location number, respectively. 
表 3 北部冬麦区小麦品种试验在不同遗传力水平下需要的重复数和试点数量定量分析

Table 3 Replicate and test location number needed to achieve different heritability levels for regional winter wheat variety trials in Northern winter wheat region

\begin{tabular}{|c|c|c|c|c|c|c|c|c|c|c|c|c|c|}
\hline \multirow{2}{*}{$\begin{array}{l}\text { 试验因素 } \\
\text { Trial factor }\end{array}$} & \multirow{2}{*}{$\begin{array}{l}\text { 组别 } \\
\text { Group }\end{array}$} & \multirow{2}{*}{$\begin{array}{c}\text { 信噪比 } \\
Q_{\mathrm{r}} / Q_{\mathrm{e}}\end{array}$} & \multirow{2}{*}{$\begin{array}{l}\text { 数量 } \\
\text { No. }\end{array}$} & \multicolumn{10}{|c|}{$\begin{array}{l}\text { 不同遗传力 }(H) \text { 水平下需要的重复数和试点数 }{ }^{\mathrm{N}} \\
\text { Replicate and test location needed at different heritabilities }(H)^{\mathrm{N}}\end{array}$} \\
\hline & & & & 0.65 & 0.75 & 0.80 & 0.83 & 0.85 & 0.86 & 0.87 & 0.88 & 0.90 & 0.95 \\
\hline 重复 & 水地 Irrigated & 0.53 & 3 & 1.0 & 1.6 & 2.1 & 2.6 & 3.0 & 3.3 & 3.5 & 3.9 & 4.8 & 10.1 \\
\hline \multirow[t]{2}{*}{ Replicate } & 旱地 Rainfed & 0.39 & 3 & 0.7 & 1.2 & 1.6 & 1.9 & 2.2 & 2.4 & 2.6 & 2.9 & 3.5 & 7.4 \\
\hline & 北部冬麦区 NWWR & 0.46 & 3 & 0.9 & 1.4 & 1.8 & 2.2 & 2.6 & 2.8 & 3.1 & 3.4 & 4.1 & 8.7 \\
\hline \multirow{3}{*}{$\begin{array}{c}\text { 试点 } \\
\text { Location }\end{array}$} & 水地 Irrigated & 3.63 & 11 & 6.7 & 10.9 & 14.5 & 17.7 & 20.6 & 22.3 & 24.3 & 26.6 & 32.7 & 69.0 \\
\hline & 旱地 Rainfed & 4.17 & 9 & 7.7 & 12.5 & 16.7 & 20.4 & 23.6 & 25.6 & 27.9 & 30.6 & 37.5 & 79.2 \\
\hline & 北部冬麦区 NWWR & 3.90 & 10 & 7.2 & 11.7 & 15.6 & 19.0 & 22.1 & 24.0 & 26.1 & 28.6 & 35.1 & 74.1 \\
\hline
\end{tabular}

重复的信噪比 $\left(Q_{\mathrm{r}}\right)$ 为单年单点试验的平均值; 试点的信噪比 $\left(Q_{\mathrm{e}}\right)$ 一年多点试验校正平均值; 合格数量为 $2010-2019$ 年期间北部冬麦区 水地组和旱地组实际汇总的重复或试点数量。

The noise-signal ratio of replicate $\left(Q_{\mathrm{r}}\right)$ is the mean of all single trials in each group, while location $Q_{\mathrm{e}}$ is the mean of all one-year multi-locational trials in the group. ${ }^{N}$ is the mean of replicates or test locations summarized in the annual wheat cultivar trial report of the irrigated group and rainfed group trials in Northern winter wheat region (NWWR) from 2010 to 2019.

北部冬麦区小麦品种试验需要的试点数量分别约为 11 个、 13 个和 12 个; 水地组、旱地组及北部冬麦 区小麦品种试验平均汇总的试点数分别约为 11 个、 9 个和 10 个, 其试验遗传力分别达到了约 $0.75 、 0.68$ 和 0.71 的水平, 水地组已经符合小麦品种试验的精 确度要求, 而旱地组需要增加 4 个试点才可以达到 试验的精确度要求; 在条件许可的情况下, 将旱地 组试点数增加到 13 个, 可将整体试验的遗传力水平 提升到 0.75 ; 如需将遗传力提高到 0.85 水平, 则水 地组和旱地组分别需要约 21 个和 24 个试点; 如需 将遗传力提高到 0.90 水平, 则水地组和旱地组分别 需要约 33 个和 38 个试点, 其后继续增加试点数没 有意义。

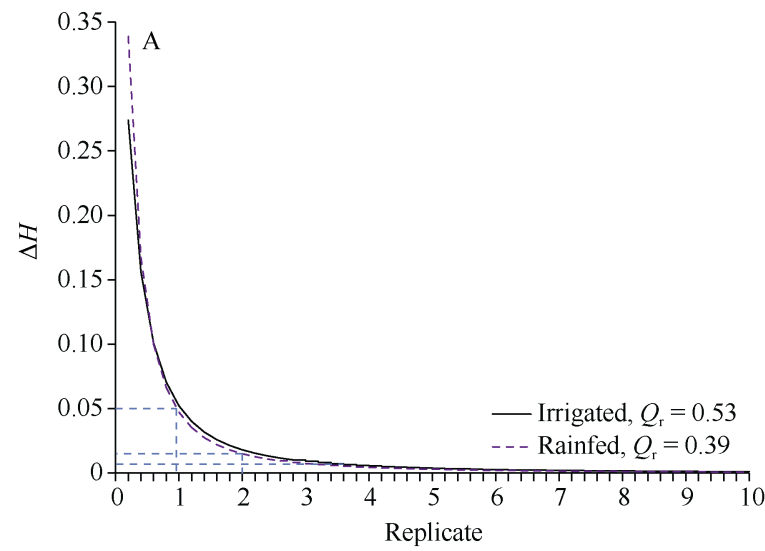

\section{3 小麦品种试验中重复数和试点数对遗传力 增量的影响}

依据 2010-2019年北部冬麦区水地组和旱地组 小麦品种试验的单点试验平均信噪比 $\left(Q_{\mathrm{r}}\right)$ 和一年多 点试验平均信噪比 $\left(Q_{\mathrm{e}}\right)$ 与重复次数或试点数及遗传 力的关系式，计算在设定区间内与单位增量的重复 次数和试点数量相对应的遗传力增量。图 2 表明, (1)水 地组和旱地组小麦品种试验重复数和试点数增量对 遗传力增量的影响趋势基本一致, 可合并分析。(2)重 复次数以 0.2 为单位, 在区间 $[0,1]$ 中每增加一个单位, 遗传力依次提升 $0.30 、 0.16 、 0.10 、 0.07$ 和 0.05 , 当 重复次数为 1 时遗传力达到 0.68 ; 在区间 $[1,5]$ 中每 增加一次重复, 遗传力依次提升 $0.13 、 0.05 、 0.03$

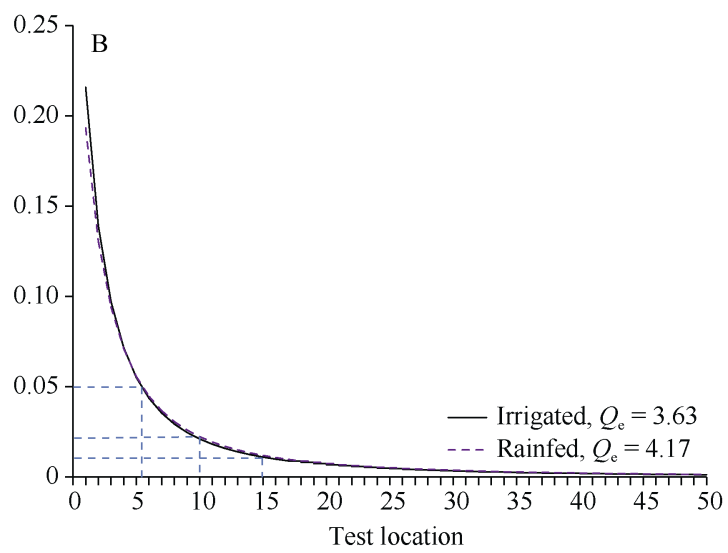

图 2 北部冬麦区小麦品种试验的遗传力增量 $(\Delta H)$ 与单位重复数或试点数的关系

Fig. 2 Relationship between heritability increment $(\Delta H)$ and per unit replicates or test location number for regional winter wheat variety trials in Northern winter wheat region 
和 0.02 ; 其后继续增加重复数, 遗传力基本不再提 升。重复数从 1 次增加到 2 次、 3 次和 4 次, 遗传力依 次从 0.68 提升到 $0.81 、 0.86 、 0.89$ 和 0.91 ，可见，第 2 次重复的遗传力增量较大, 第3次重复的遗传力增 加已经很小, 后续重复数增加对遗传力的提升效应 十分微小。(3)试点数以 1 为单位，在区间 $[1,5]$ 中每增 加 1 个试点, 遗传力增量依次为 $0.20 、 0.13 、 0.10 、 0.07$ 和 0.06 , 从 0.20 提高到 0.60 ; 其后继续增加试点数, 遗传力的提升速度减缓, 在试点数区间 $[6,10]$ 中每增 加 1 个试点约提高遗传力 0.02 , 在试点数区间 $[11,15]$ 中每增加 1 个试点约提高遗传力 0.01 , 其后试点数增 加对遗传力的提升效应较小。试点数从 5 个增加到 10 个、 15 个和 20 个, 遗传力依次从 0.56 提升到 $0.72 、 0.79$ 和 0.84 , 后续试点数增加对遗传力的提升效应基本 上没有意义。

\section{3 讨论}

\section{1 小麦品种区域试验效率的评价指标探讨}

农作物多环境品种试验 (品种区域试验) 是客观 评价农作物品种的丰产性、稳产性和适应性及生产 应用价值必要环节 ${ }^{[10]}$ 。作物区域试验的科学性、可 靠性和试验效率主要决定于其对品种间差异的鉴别 能力, 而鉴别力又决定于试验的精确度 ${ }^{[11]}$ 。试验精 确度在区域试验质量评价中一直受到广泛关注 ${ }^{[12-16]}$ 。 试验的精确度主要与试验误差有关, 试验误差越大, 则精确度越低。区域试验中通常用试验误差变异系 数 $(\mathrm{CV}$, coefficient of error variation)来表示试验的精 确度, 并将 CV大于 $15 \%$ 的单年单点试验数据在汇总 报告中剔除。作为更直接的试验对品种鉴别能力的 指标, 孔繁玲等 ${ }^{[11]}$ 将品种间多重比较时的最小显著 差数(least significant difference, LSD)与总体均值的 比率称为相对最小显著差数(relative least significant difference, RLSD)作为试验精确度的指标 ${ }^{[17]}$ 。然而, 一年多点品种区域试验的精确度和有效性不仅受到 遗传效应和误差大小的影响, 还受到品种与环境互 作效应等因素的影响 ${ }^{[18-20]}$ 。一组由多个试验误差变 异都很小的单点试验组成一年多点品种区域试验并 不一定是有效的试验。例如, 2017年北部冬麦区旱地 组小麦区域试验中全部 9 个单点试验中的 $\mathrm{CV}$ 值都在 $8 \%$ 以下, 多点联合方差分析的 CV 值仅为 $3.8 \%$, 但 其试验遗传力却只有 0.30 。从 $\mathrm{CV}$ 值来看这是一个精 确度很好的试验, 而从遗传力来看又是一个“坏试 验”, 因为它对品种的鉴别能力太差, 无法分辨品种
间的真实遗传差异。品种与环境互作方差大而遗传 方差相对很小是产生这种现象的主要原因。当遗传 力接近于 0 时, 无论品种间表型差异有多大, 基于表 型变异的品种选择都是完全无效的 ${ }^{[8]}$ 。遗传力表达 了试验对品种间遗传差异的鉴别能力和对品种的选 择效率, 也就是体现了品种区域试验的效率。在农 作物品种多环境试验质量评价体系中除了用 $\mathrm{CV}$ 表 达试验的误差控制水平外, 还应当将试验遗传力作 为试验有效性的重要评价指标 ${ }^{[5]}$ 。Yan等 ${ }^{[6]}$ 研究发现 $H=0.75$ 是比较适当的试验评价标准。许乃银等 ${ }^{[5]}$ 研 究证实了遗传力保持 0.75 的水平是我国棉花品种区 域试验质量评价比较适合的标准，但也需要依据具 体品种试验发展水平确定其适宜的目标遗传力水 平。本研究发现我国北部冬麦区小麦品种试验水地 组和旱地组在正常年份的试验遗传力平均分别为 0.75 和 0.68 ，同时也考虑到小麦品种区域试验对品 种推荐审定和应用十分重要, 每年都可能有部分试 验点因为各种异常原因而报废, 为充分保证试验汇 总结果的可靠性, 可按遗传力为 0.85 的水平需求安 排试验点数量和重复次数。

\section{2 小麦品种区域试验重复次数和试点数量的} 优化策略

小麦品种区域试验从试验设计、田间管理和试 验统计分析等各项工作都是为了提高小麦品种试验 的遗传力, 从而提高试验的品种选择效率 ${ }^{[5]}$ 。品种 多点试验的遗传力受到试验误差、品种与试点互作 效应、试点数量和试验重复次数等因素的综合影响。 因此, 加强试验田间管理以减少试验误差、开展适 当的品种生态区划分以减少品种与环境互作效应、 适当增加试验重复次数和试点数量区域试验等措施 有利于提高试验遗传力和试验效率。试验遗传力随 着重复次数和试点数量增加而提高的速率符合先快 后慢的“边际效应递减”规律, 在达到适当的数量后 再增加重复次数和试点数量对遗传力的增量贡献很 小, 试验效率提高不大。同时, 重复次数和试点数 量的增加也意味着试验投入的人力、物力和财力成 本的增加，成本投入对试验效率的提高符合报酬递 减规律。因此, 品种区域试验重复次数和试点数量 的优化就是依据遗传力随着重复次数和试点数量增 加的递增规律, 设计适当的重复次数和试点数量在 充分保证试验的可靠性和科学性的前提下又能节省 试验成本, 减少不必要的资源浪费。严威凯等 ${ }^{[6]}$ 研 究指出遗传力达到 0.75 的水平是试验成本和试验效 
率的产投比“拐点”, 遗传力大于 0.75 时的试点数量 增加对遗传力增量效应将快速衰退。本研究也证实 了试验遗传力随着重复次数和试点数的增加表现为 先快后慢的曲线关系, 遗传力在 0.75 以下时表现为 “快速增长期”, 在 0.75 和 0.90 期间表现为“缓慢增 长期”, 在 0.90 之后表现为“增长停滞期”。本研究发 现我国北部冬麦区小麦品种试验第 2 次重复的遗传 力增量较大, 第 3 次重复的遗传力增加已经很小, 后续重复数增加对遗传力的提升效应十分微小; 试 点数从 5 个增加到 10 个、 15 个和 20 个, 遗传力依 次从 0.56 提升到 $0.72 、 0.79$ 和 0.84 , 后续试点数增 加对遗传力的提升效应基本上没有意义。因此, 北 部冬麦区小麦品种试验可保持当前的 3 次重复; 水 地组的试点数量可保持在 11 个左右; 旱地组可将试 点数增加到 13 个, 使遗传力达到 0.75 ; 在条件许可 的前提下, 旱地组和水地组的试点数可分别增加到 15 个和 17 个, 使遗传力达到 0.80 的水平。

\section{4 结论}

我国北部冬麦区小麦品种试验采用 3 次重复可 以充分保证试验遗传力和试验效率符合试验质量要 求, 目前设置的试点数量也可以充分满足区域试验 的精确度要求。针对小麦品种试验重复次数和试点 数量的优化方案包括,重复次数仍可设计为 3 次; 水 地组可保持当前 11 个左右的试点数量, 旱地组和在 国家财力、物力和人力等条件许可的情况下，可以 将试点数量增加到 13 个左右, 从而使北部冬麦区小 麦品种试验遗传力达到到 0.75 的水平; 如需将遗传 力提高在 0.80 水平, 则需要约 16 个试点; 增加的试 点数量可与增加重复次数相比较, 优先选择增加小 区数量较少的方案, 有利于节约试验成本。

\section{References}

[1] 胡学旭, 孙丽娟, 周桂英, 吴丽娜, 陆伟, 李为喜, 王爽, 杨秀 兰, 宋敬可, 王步军. 2000-2015 年北部、黄淮冬麦区国家区试 品种的品质特征. 作物学报, 2017, 43: 501-509.

Hu X X, Sun L J, Zhou G Y, Wu L N, Lu W, Li W X, Wang S, Yang X L, Song J K, Wang B J. Quality characteristics of winter wheat varieties tested in national trials in northern region and Yellow-Huai River valley winter wheat zone from 2000 to 2015. Acta Agron Sin, 2017, 43: 501-509 (in Chinese with English abstract).

[2] 赵广才. 小麦优质高产栽培理论与技术. 北京: 中国农业科学 技术出版社, 2018.

Zhao G C. Good Quality and High Yield Cultivation of Wheat: Theory and Technology. Beijing: China Agricultural Science and Technology Press, 2018.
[3] 孟繁圆, 冯利平, 张丰瑶, 张神, 伍露, 王春雷, 问锦涛, 彭明 喜, 莫志鸿, 余卫东. 北部冬麦区冬小麦越冬冻害时空变化特 征. 作物学报, 2019, 45: 1576-1585.

Meng F Y, Feng L P, Zhang F Y, Zhang W, Wu L, Wang C L, Yan J T, Peng M X, Mo Z H, Yu W D. Temporal and spatial variations of winter wheat freezing injury in northern winter wheat region. Acta Agron Sin, 2019, 45: 1576-1585 (in Chinese with English abstract).

[4] 胡学旭, 孙丽娟, 周桂英, 吴丽娜, 陆伟, 李为喜, 王爽, 杨秀 兰, 宋敬可, 王步军. 2000-2015 年国家黄淮和北部冬麦区域 试验品种品质分析. 中国农业科学, 2016, 49: 4677-4686.

Hu X X, Sun L J, Zhou G Y, Wu L N, Lu W, Li W X, Wang S, Yang X L, Song J K, Wang B J. Quality variation of national tested varieties in northern winter wheat region and Yellow-Huai River valley winter wheat region from 2000 to 2015. Sci Agric Sin, 2016, 49: 4677-4686 (in Chinese with English abstract).

[5] 许乃银, 金石桥, 李健. 我国棉花品种区域试验重复次数和试 点数量的设计. 作物学报, 2016, 42: 43-50.

Xu N Y, Jin S Q, Li J. Design of test location number and replicate frequency in the regional cotton variety trials in China. Acta Agron Sin, 2016, 42: 43-50 (in Chinese with English abstract)

[6] Yan W K, Frégeau-Reid J, Martin R, Pageau D, Mitchell-Fetch J. How many test locations and replications are needed in crop variety trials for a target region? Euphytica, 2015, 202: 361-372.

[7] 翟虎渠. 应用数量遗传. 北京: 中国农业出版社, 2001.

Zhai H Q. Applied Quantitative Genetics. Beijing: Chinese Agriculture Press, 2001 (in Chinese).

[8] Yan W K. Crop Variety Trials: Data Management and Analysis. Oxford, UK: Wiley-Blackwell, 2014.

[9] Yan W K. GGEbiplot-A windows application for graphical analysis of multienvironment trial data and other types of two-way data. Agron J, 2001, 93: 1111-1118.

[10] 许乃银, 李健. 我国棉花品种区域试验精确度的演变分析. 棉 花学报, 2016, 28: 34-41

Xu N Y, Li J. Evolution analysis of the precision of regional cotton variety trials in China. Cotton Sci, 2016, 28: 34-41 (in Chinese with English abstract).

[11] 孔繁玲, 张群远, 杨付新, 郭恒敏. 棉花品种区域试验的精确 度探讨. 作物学报, 1998, 24: 601-607.

Kong F L, Zhang Q Y, Yang F X, Guo H M. Studies on the precision of regional cotton variety trial. Acta Agron Sin, 1998, 24: 601-607 (in Chinese with English abstract).

[12] 许乃银, 金石桥. 棉花品种区域试验适宜试验点数量的抽样 估计. 棉花学报, 2013, 25: 57-62.

Xu N Y, Jin S Q. Sampling estimation of suitable quantity of test sites in cotton variety regional trials. Cotton Sci, 2013, 25: 57-62 (in Chinese with English abstract).

[13] Gouy M, Rousselle Y, Bastianelli D, Lecomte P, Bonnal L, Roques D, Efile J C, Rocher S, Daugrois J, Toubi L, Nabeneza S, Hervouet C, Telismart H, Denis M, Thong-Chane A, Glaszmann J C, Hoarau J Y, Nibouche S, Costet L. Experimental assessment of the accuracy of genomic selection in sugarcane. Theor Appl Genet, 2013, 126: 2575-2586.

[14] Iwata H, Jannink J. Accuracy of genomic selection prediction in barley breeding programs: a simulation study based on the real 
single nucleotide polymorphism data of barley breeding lines. Crop Sci, 2011, 51: 1887-1902.

[15] Kelly A M, Smith A B, Eccleston J A, Cullis B R. The accuracy of varietal selection using factor analytic models for multi-environment plant breeding trials. Crop Sci, 2007, 47: 1063-1070.

[16] Gauch H G, Zobel R W. Accuracy and selection success in yield trial analyses. Theor Appl Genet, 1989, 77: 473-481.

[17] 王洁, 廖琴, 胡小军, 万建民. 北方稻区国家水稻品种区域试 验精确度分析. 作物学报, 2010, 36: 1870-1876.

Wang J, Liao Q, Hu X J, Wan J M. Precision evaluation of rice variety regional trials in Northern China. Acta Agron Sin, 2010,
36: 1870-1876 (in Chinese with English abstract).

[18] Brancourt-Hulmel M, Lecomte C. Effect of environmental variates on genotype $\times$ environment interaction of winter wheat: a comparison of biadditive factorial regression to AMMI. Crop Sci, 2003, 43: 608-617.

[19] Brennan P S, Byth D E, Drake D W, DeLacy I H, Butler D G. Determination of the location and number of test environments for a wheat cultivar evaluation program. Aust J Agr Res, 1981, 32: 189-201.

[20] Imrie B C, Drake D W, Delacy I H, Byth D E. Analysis of genotypic and environmental variation in international mungbean trials. Euphytica, 1981, 30: 301-311. 